\title{
A multicenter clinical study to determine the feasible initial dose of lenalidomide for maintenance therapy in patients with multiple myeloma following autologous peripheral blood stem-cell transplantation
}

\author{
MASAHIDE YAMAMOTO $^{1^{*}}$, KAZUTERU OHASHI $^{2 *}$, KAZUHIKO KAKIHANA $^{2 *}$ YUUICHI NAKAMURA $^{3 *}$, \\ TAKUYA KOMENO $^{4 *}$, HIROSHI KOJIMA $^{5^{*}}$, SATOSHI MORITA $^{6^{*}}$ and HISASHI SAKAMAKI ${ }^{2 *}$ \\ ${ }^{1}$ Department of Hematology, Tokyo Medical and Dental University, Tokyo 113-0034; ${ }^{2}$ Hematology Division, \\ Tokyo Metropolitan Cancer and Infectious Diseases Center, Komagome Hospital, Tokyo 113-8677; ${ }^{3}$ Department \\ of Hematology, Saitama Medical University Hospital, Iruma, Saitama 350-0495; ${ }^{4}$ Department of Hematology, \\ Mito Medical Center, National Hospital Organization, Mito, Ibaraki 311-3193; ${ }^{5}$ Department of Clinical Oncology, \\ Ibaraki Prefectural Central Hospital, Ibaraki Clinical Education and Training Center, University \\ of Tsukuba Hospital, Tsukuba, Ibaraki 305-8576; ${ }^{6}$ Biomedical Statistics and Bioinformatics, \\ Graduate School of Medicine, Kyoto University, Kyoto 606-8502, Japan
}

Received November 10, 2015; Accepted March 13, 2016

DOI: $10.3892 / \mathrm{mco} .2016 .833$

\begin{abstract}
Maintenance therapy with lenalidomide (LEN) for patients with multiple myeloma (MM) following autologous peripheral blood stem cell transplantation (auto-PBSCT) may be a promising option for preventing relapse or disease progression. However, the recommended dose of LEN has yet to be firmly established. We herein report the results of a multicenter clinical study for determining the feasible initial dose (FID) of LEN. In this trial, a total of 11 patients who achieved a very good partial response or complete response following auto-PBSCT were enrolled from five transplant centers in Japan. Three dose levels of LEN (level 0, $5 \mathrm{mg}$; level 1, $10 \mathrm{mg}$; and level 2,:15 mg) were tested in this study. FID was defined as the maximum estimated dose at which $70 \%$ of the patients could receive maintenance therapy for 12 weeks without any serious adverse events or disease progression. Using a continual reassessment method, 6 patients were assigned to level 0 and the remaining 5 patients were assigned to level 1 . All 6 patients $(100 \%)$ at level 0 , but only 2 patients $(40 \%)$ at level 1, completed 12 weeks of administration with their assigned dose of LEN. The results of our study demonstrated
\end{abstract}

Correspondence to: Dr Masahide Yamamoto, Department of Hematology, Tokyo Medical and Dental University, 1-5-45 Yushima, Bunkyo-Ku, Tokyo 113-0034, Japan

E-mail: hide.hema@tmd.ac.jp

* On behalf of the Ochanomizu Hematology Study Group

Key words: lenalidomide, dose-finding study, adverse effect, autologous peripheral blood stem-cell transplantation that, although $5 \mathrm{mg}$ of LEN was acceptable in terms of safety, $7.5 \mathrm{mg}$ of LEN may also be an acceptable FID.

\section{Introduction}

The prognosis of multiple myeloma (MM) has been significantly improved by the recent introduction of several novel agents, such as thalidomide (THAL), lenalidomide (LEN) and bortezomib (BOR) (1). The use of these agents, particularly in younger patients, prior to or following autologous peripheral stem cell transplantation (auto-PBSCT), may result in improved transplant outcomes, with a higher very good partial response (VGPR) or complete response (CR) rate, which may eventually translate into prolonged progression-free survival (PFS) and/or overall survival (OS) $(2,3)$. Regarding maintenance therapy, the results of several recent studies have suggested use of these agents as a promising option for preventing relapse or disease progression after auto-PBSCT (4-7). However, THAL may not be suitable as a long-term maintenance agent, mainly due to its neurotoxic side effect profile $(4,5)$.

Conversely, LEN is less toxic and may be more feasible for use in a maintenance setting, as the results from several studies have demonstrated its effects in terms of improved outcomes and prolonged PFS after auto-PBSCT.

The results of a recent randomized, prospective trial support the rationale for the use of LEN after auto-PBSCT (7). Based on the aforementioned results, more patients are currently undergoing LEN maintenance therapy following auto-PBSCT; however, the recommended dose of LEN for maintenance has yet to be firmly established. Currently, LEN is administered at $5-15 \mathrm{mg} /$ day for maintenance. We herein report the results of a multicenter clinical study for determining the feasible initial dose (FID) of LEN for maintenance therapy in patients with MM following auto-PBSCT. 


\section{Patients and methods}

Study participants. Patients with MM, aged 15-70 years, who achieved VGPR or a better response after auto-PBSCT, were enrolled in this trial. The main inclusion criteria were a good performance status (0-2), an absence of renal dysfunction as assessed by creatinine clearance $>60 \mathrm{ml} / \mathrm{min}$, an absence of deep vein thrombosis or pulmonary thromboembolism, and recovery from myelosuppression following auto-PBSCT. Female patients were either postmenopausal, surgically sterilized, or willing to use an acceptable method of birth control for the entire duration of the study. Likewise, male patients consented to using an acceptable method of contraception for the entire duration of the study. The main exclusion criteria included grade $\geq 2$ peripheral neuropathy, active infection, positive serology for human immunodeficiency virus or hepatitis B or C viruses, pregnancy and breast-feeding. This trial was approved by the Institutional Ethics Committee at each participating institution and registered as UMIN000012700. Written informed consent was obtained from all the patients prior to registration in accordance with the Declaration of Helsinki.

Treatment schedule. The patients required LEN maintenance therapy within 6 months of undergoing auto-PBSCT. The three selected dose levels of LEN (level 0,5 mg; level 1, $10 \mathrm{mg}$; and level $2,15 \mathrm{mg}$ ) were determined based on previously published data (6-8). The assigned dose of LEN was administered orally once per day for 12 weeks, without any drug holidays. The patients were able to continue receiving LEN during the scheduled period, unless a dose-limiting toxicity (DLT) occurred, obvious evidence of disease progression appeared, or the patient refused to continue treatment.

Assessment of treatment toxicity and efficacy. According to the National Cancer Institute Common Toxicity Criteria version 4.0 (http://ctep.cancer.gov/protocolDevelopment/electronic_applications/ctc.htm\#ctc_40), any hematological or non-hematological adverse event (AE) of grade $\geq 3$, or discontinuation of drug administration due to any grade of AEs directly attributable to LEN, were considered as DLTs. The assessment of disease response was based on the International Uniform Response Criteria (9).

Study design and statistical analysis. The FID was estimated using the continual reassessment method (CRM) $(10,11)$. Dose escalations or de-escalations for consecutive patient cohorts and the size of each cohort were based on the dose-finding CRM algorithm and clinical judgment. Jumping from level 0 to 2 was not permissible in the CRM algorithm. The target completion rate of maintenance therapy was set at $70 \%$. The FID of this trial was determined as the dose that was closest to the level at which $70 \%$ of patients would complete 12 weeks of maintenance therapy without experiencing a severe AE. We determined the starting dose in this trial to be level 1 , and the first 3 patients were treated at this level. According to the pre-specified dose de-escalation rule, if DLT was observed in 2 of the first 3 patients, another 3 patients allocated to the second cohort were treated at level 0 . This phase I study was expected to require a project sample-size of $\leq 15$ patients.

\section{Results}

Patient characteristics. Between August, 2011 and April, 2014, a total of 11 patients ( 8 in CR and 3 in VGPR) from five transplant centers in Japan were enrolled in this study, with a median of 99 days (range, 60-168 days) after auto-PBSCT. Using the CRM algorithm, 6 patients were assigned to level 0 and 5 were assigned to level 1 (Fig. 1). The characteristics of these 11 patients are summarized in Table I. The median age was 58 years (range, 41-65 years), and 7 patients were men (64\%). The subtypes of monoclonal immunoglobulin (Ig) detected in the 11 patients were $\operatorname{IgG}(n=4), \operatorname{IgA}(n=4), \operatorname{IgD}(n=1)$ and light-chain-only disease $(n=2)$. All the patients received a BOR-containing induction regimen prior to auto-PBSCT; however, no patients received prior THAL.

Toxicity. The time-dependent changes in hematological and non-hematological AEs observed during maintenance therapy with LEN are detailed in Table II. Of the 6 patients assigned to level 0, $3(50 \%)$ experienced neutropenia (grade $\leq 2$ ) at approximately 8 weeks after initiating LEN maintenance therapy; however, all the patients spontaneously recovered from neutropenia despite continuous administration of LEN. As for the remaining patients, no serious AEs (SAEs) were observed at level 0 . As shown in Table II, more patients experienced AEs at level 1. One patient [unique patient number (UPN) 3]developed pneumonia as a consequence of neutropenia in week 10 of treatment; therefore, LEN administration was discontinued at 11 weeks. Another patient (UPN 2) developed both neutropenia and thrombocytopenia in addition to peripheral neuropathy in week 4 of treatment; although this patient's dose was reduced to $5 \mathrm{mg}$ (level 0), thrombocytopenia progressed and LEN administration was discontinued soon after. Another patient (UPN 9) developed liver dysfunction in week 2 of the treatment, which had progressed to grade 2 by week 6 . Administration of LEN was discontinued for this patient as well. Grade 3/4 toxicities were not observed in any of the study patients.

Dose escalation and de-escalation. The dose-finding CRM algorithm and clinical judgment used in this study are shown in Fig. 1. Of the first 3 patients assigned to level 1,2 patients (UPN 2 and 3) encountered DLT [discontinuation of LEN with the attending physician's judgment based on progressive grade 2 hematological $(n=1)$ and non-hematological $(n=1)$ AEs]. According to the pre-specified dose de-escalation rules for this trial, the dose level for the second patient cohort was de-escalated to level 0 . However, none of the 3 patients (UPN 4, 5 and 6) at level 0 in the second cohort experienced any DLTs. In the third cohort, 1 patient (UPN 7) remained assigned to level 0, but did not experience any DLTs. Therefore, the next 2 patients (UPN 8 and 9) were assigned to level 1 in the third cohort, although 1 (UPN 9) of the 2 patients again experienced a DLT (discontinuation of LEN administration occurred with the attending physician's judgment based on a progressive grade 2 non-hematological AE). Consequently, the estimated level 1 completion rate was considerably lower compared with the target level, and the next patient cohort was treated at level 0 . Of the subsequent 2 patients (UPN 10 and 11) treated at level 0 , as the fourth cohort, neither experienced DLTs. Based on the 
Table I. Characteristics of the patients who received maintenance therapy with two dose levels of lenalidomide.

\begin{tabular}{|c|c|c|}
\hline Characteristics & $\begin{array}{l}\text { Level } 0(5 \mathrm{mg}) \\
\qquad \mathrm{n}=6\end{array}$ & $\begin{array}{l}\text { Level } 1(10 \mathrm{mg}) \\
\qquad \mathrm{n}=5\end{array}$ \\
\hline Gender (male/female) & $5 / 1$ & $2 / 3$ \\
\hline Median age, years (range) & $59(41-65)$ & $57(46-63)$ \\
\hline \multicolumn{3}{|l|}{ Performance status } \\
\hline $0-1$ & 3 & 5 \\
\hline 2 & 3 & 0 \\
\hline \multicolumn{3}{|l|}{ Types of immunoglobulin } \\
\hline $\operatorname{IgG}$ & 3 & 1 \\
\hline $\operatorname{Ig} \mathrm{A}$ & 2 & 2 \\
\hline $\operatorname{IgD}$ & 0 & 1 \\
\hline BJP & 1 & 1 \\
\hline \multicolumn{3}{|l|}{ International staging system } \\
\hline Stage I & 2 & 0 \\
\hline Stage II & 3 & 3 \\
\hline Stage III & 1 & 2 \\
\hline \multicolumn{3}{|l|}{ Durie-Salmon staging system } \\
\hline Stage I & 1 & 0 \\
\hline Stage II & 1 & 1 \\
\hline Stage III & 4 & 4 \\
\hline Durie-Salmon sub-classifications (A/B) & $0 / 6$ & $4 / 1$ \\
\hline \multicolumn{3}{|l|}{ Symptoms related to myeloma } \\
\hline Hypercalcemia & 2 & 4 \\
\hline Renal insufficiency & 0 & 2 \\
\hline Anemia & 5 & 4 \\
\hline Bone disease & 4 & 4 \\
\hline \multicolumn{3}{|l|}{ Initial therapy } \\
\hline VAD-BD & 2 & 2 \\
\hline BD & 2 & 3 \\
\hline VCD & 2 & 0 \\
\hline \multicolumn{3}{|l|}{ Best response after auto-PBSCT } \\
\hline $\mathrm{CR}^{\mathrm{a}}$ & 4 & 4 \\
\hline VGPR $^{b}$ & 2 & 1 \\
\hline $\begin{array}{l}\text { Median duration from auto-PBSCT } \\
\text { to maintenance therapy (range) }\end{array}$ & 95 days (60-121) & 99 days (68-168) \\
\hline
\end{tabular}

aPatients with CR must have no abnormal monoclonal proteins present in their serum or urine. In addition, any soft tissue plasmacytomas must

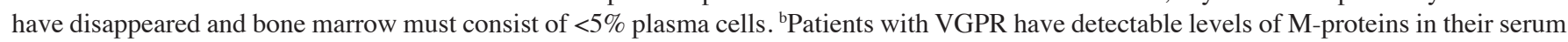
and urine when immunofixation is used, but not electrophoresis. Alternatively, VGPR is defined as patients with a $\geq 90 \%$ reduction in their serum M-component, with <100 mg of urinary M-component over a period of $24 \mathrm{~h}$. BJD, Bence-Jones protein; VAD, vincristine, doxorubicin and dexamethasone; BD, bortezomib and dexamethasone; VCD, bortezomib, cyclophosphamide and dexamethasone; CR, complete response; VGPR, very good partial response; auto-PBSCT, autologous peripheral blood stem cell transplantation.

results of the sensitivity analysis and the observed toxicities at each dose level, we concluded that LEN maintenance therapy at level 0 was acceptable in terms of safety.

Response to LEN maintenance. The disease status prior to and following LEN maintenance therapy is indicated in Table III. The results from the 8 assessable patients who completed 12 weeks of LEN maintenance therapy revealed no disease progression or obvious response. The 2 patients with VGPR maintained that status and did not achieve CR. The 6 patients with CR also maintained stable disease conditions during LEN maintenance therapy.

\section{Discussion}

Accumulated clinical experience has provided convincing evidence that novel agents, such as THAL, LEN and BOR, alone or in combination, are efficacious in all stages 
Table II. Time-dependent changes of adverse events observed during lenalidomide maintenance therapy.

Time-dependent changes, grade (CTCAE version 4.0)

\begin{tabular}{|c|c|c|c|c|c|c|c|}
\hline \multirow[b]{2}{*}{ Dose level } & \multirow[b]{2}{*}{ UPN } & \multirow[b]{2}{*}{ Adverse events } & & & & & \\
\hline & & & Before & 2 weeks & 4 weeks & 8 weeks & 12 weeks \\
\hline \multirow[t]{14}{*}{ Level 0 (5 mg) } & \multirow[t]{4}{*}{4} & Liver dysfunction & 1 & 1 & 1 & 1 & 1 \\
\hline & & Thrombocytopenia & 1 & 1 & 1 & 1 & 1 \\
\hline & & Hypokalemia & 1 & 1 & 1 & 1 & 1 \\
\hline & & Hyperglycemia & - & - & - & - & 1 \\
\hline & 5 & Neutropenia & - & - & 1 & 2 & - \\
\hline & 6 & Thrombocytopenia & - & - & - & - & 1 \\
\hline & \multirow[t]{4}{*}{7} & Constipation & - & 1 & - & - & - \\
\hline & & Muscle weakness & - & - & 1 & - & - \\
\hline & & Neutropenia & - & - & - & 2 & - \\
\hline & & URTI & - & - & - & 2 & - \\
\hline & 10 & No adverse events & - & - & - & - & - \\
\hline & \multirow[t]{3}{*}{11} & Thrombocytopenia & 1 & - & - & - & - \\
\hline & & Skin rash & - & 1 & 1 & 1 & 1 \\
\hline & & Neutropenia & - & - & - & 2 & - \\
\hline \multirow[t]{12}{*}{ Level 1 (10 mg) } & \multirow[t]{4}{*}{1} & Diarrhea & - & - & - & 1 & 1 \\
\hline & & Hypothyroidism & - & - & - & - & 1 \\
\hline & & URTI & - & - & - & - & 1 \\
\hline & & Peripheral neuropathy & - & - & - & - & 1 \\
\hline & \multirow[t]{3}{*}{$2^{\mathrm{a}}$} & Thrombocytopenia & 1 & 1 & 1 & $\begin{array}{c}2 \\
\text { (at } 6 \text { weeks) }\end{array}$ & N/A \\
\hline & & Peripheral neuropathy & - & 1 & 1 & $\begin{array}{c}1 \\
\text { (at } 6 \text { weeks) }\end{array}$ & N/A \\
\hline & & Neutropenia & - & - & 2 & $\begin{array}{c}2 \\
\text { (at } 6 \text { weeks) }\end{array}$ & N/A \\
\hline & \multirow[t]{2}{*}{$3^{b}$} & Neutropenia & - & - & - & - & $\begin{array}{c}2 \\
\text { (at } 10 \text { weeks) }\end{array}$ \\
\hline & & Pneumonia & - & - & - & - & $\begin{array}{c}2 \\
\text { (at } 10 \text { weeks) }\end{array}$ \\
\hline & \multirow[t]{2}{*}{8} & Neutropenia & - & - & - & - & 1 \\
\hline & & Thrombocytopenia & - & - & - & - & 1 \\
\hline & $9^{c}$ & Liver dysfunction & - & 1 & 1 & $\begin{array}{c}2 \\
\text { (at } 6 \text { weeks) }\end{array}$ & N/A \\
\hline
\end{tabular}

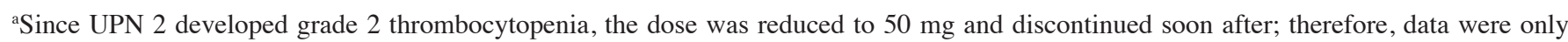
available until week 6 . ${ }^{b}$ UPN 3 suddenly developed grade 2 neutropenia at 10 weeks; therefore, lenalidomide was discontinued at 11 weeks. 'UPN 9 developed grade 1 liver dysfunction, which progressed to grade 2 at 6 weeks. Administration was discontinued at 6 weeks; therefore, data were only available until week 6. CTCAE, Common Terminology Criteria for Adverse Events; N/A, not being appreciated; UPN, unique patient number; URTI, upper respiratory tract infection.

of MM (12). Moreover, monotherapy with these agents in a maintenance setting may be a promising option for preventing relapse or disease progression after auto-PBSCT (13). However, THAL maintenance has not received general acceptance due to serious concerns regarding its neurotoxic side effects $(4,5,14,15)$. Based on the results from recent reports, the incidence of grade 3-4 peripheral neuropathy was $\leq 27 \%$, which was the main reason for discontinuing THAL maintenance $(4,5,14,15)$. It should be noted that lower doses of BOR may be used with greater tolerability and therapeutic benefits in a maintenance setting. In the HOVON-65/GMM-HD4 study, BOR maintenance achieved improved PFS as compared with THAL; however, it appeared to be less beneficial for patients who achieved at least VGPR after auto-PBSCT (16). As maintenance therapy, LEN appeared to be the most promising of the three aforementioned agents. Based on the results from two recent randomized trials (LEN maintenance vs. no maintenance), LEN appeared to be associated with improved time-to-disease progression in both studies $(6,7)$. Moreover, the beneficial effect of LEN maintenance was relevant even 
Table III. Disease status of myeloma before and after lenalidomide maintenance therapy.

Disease status

Dose level

UPN

Before starting

maintenance
At 12 weeks

of maintenance

\begin{tabular}{|c|c|c|c|}
\hline \multirow[t]{6}{*}{ Level 0 (5 mg) } & 4 & $\mathrm{CR}^{\mathrm{a}}$ & CR \\
\hline & 5 & VGPR $^{b}$ & VGPR \\
\hline & 6 & $\mathrm{CR}$ & $\mathrm{CR}$ \\
\hline & 7 & $\mathrm{CR}$ & $\mathrm{CR}$ \\
\hline & 10 & $\mathrm{CR}$ & $\mathrm{CR}$ \\
\hline & 11 & VGPR & VGPR \\
\hline \multirow[t]{5}{*}{ Level 1 (10 mg) } & 1 & CR & $\mathrm{CR}$ \\
\hline & 2 & $\mathrm{CR}$ & $\begin{array}{c}\text { N/A because of } \\
\text { discontinuation at } 6 \text { weeks }\end{array}$ \\
\hline & 3 & VGPR & $\begin{array}{c}\text { N/A because of } \\
\text { discontinuation at } 10 \text { weeks }\end{array}$ \\
\hline & 8 & $\mathrm{CR}$ & $\mathrm{CR}$ \\
\hline & 9 & $\mathrm{CR}$ & $\begin{array}{c}\text { N/A because } \\
\text { of discontinuation at } 6 \text { weeks }\end{array}$ \\
\hline
\end{tabular}

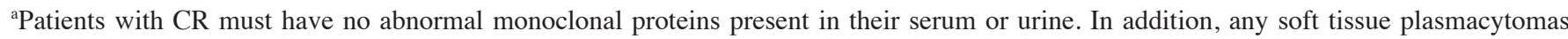
must have disappeared and bone marrow must consist of $<5 \%$ plasma cells. ${ }^{b}$ Patients with VGPR have detectable levels of M-proteins in their serum and urine when immunofixation is used, but not electrophoresis. Alternatively, VGPR is defined as patients with a $\geq 90 \%$ reduction in their serum M-component, with $<100 \mathrm{mg}$ of urinary M-component over a period of $24 \mathrm{~h}$. UPN, unique patient number; CR, complete response; VGPR, very good partial response; N/A, not being appreciated.

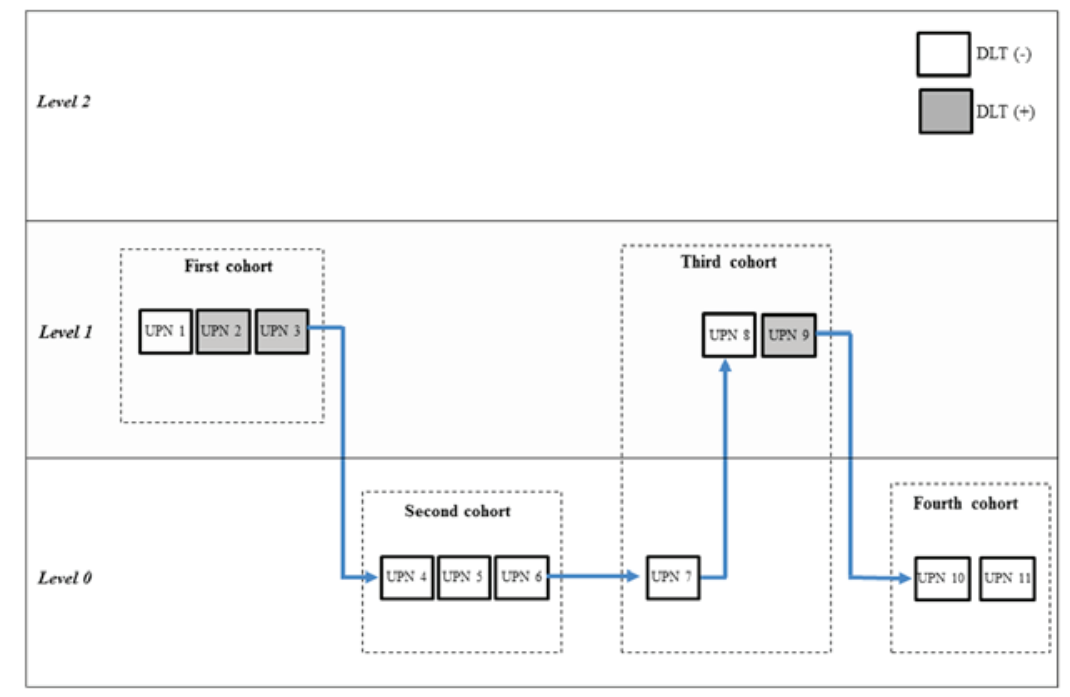

Figure 1. The dose-finding continual reassessment method (CRM) algorithm. The first cohort was assigned to level 1, but 2 out of 3 subjects encountered a dose-limiting toxicity (DLT). According to the pre-specified dose de-escalation rules of this trial, the dose level for the second cohort was de-escalated to level 0 . None of the 3 patients in the second cohort experienced DLT. In the third cohort, 1 patient was still assigned to level 0 , but did not experience any DLTs. Therefore, the next 2 patients were assigned to level 1 in the third cohort, but 1 patient experienced DLT. Consequently, the estimated level 1 completion rate was probably considerably lower than the target level, and the next subject cohort was treated at level 0 . None of the subsequent 2 patients treated at level 0 experienced any DLTs. UPN, unique patient number.

in patients who achieved VGPR/CR after auto-PBSCT in the IFM 05-02 trial (7). Although LEN appeared to be the most promising maintenance agent among the three mentioned, the dosage or duration of LEN maintenance therapy had not been formally determined in a phase I dose escalation trial. Attal et al evaluated LEN for maintenance therapy following auto-PBSCT (administered at $10 \mathrm{mg}$ per day for the first 3 months and increased up to $15 \mathrm{mg}$ if tolerated), and found 
that PFS improved from $35 \%$ in the placebo arm to $68 \%$ in the LEN arm. Unfortunately, approximately half of the patients in the LEN arm experienced grade 3-4 myelosuppression during the entire maintenance phase (7). Palumbo et al also investigated the use of LEN after auto-PBSCT (17); in their trial, LEN maintenance therapy was administered at $10 \mathrm{mg}$ for 21 days per 28-day period, and the incidence of grade 3-4 myelosuppression decreased to $23 \%$ (17).

To the best of our knowledge, this is the first study to evaluate the maintenance dose of LEN following auto-PBSCT. Although no grade 3-4 SAEs were observed in any cohort in our series, 3 patients (60\%) assigned to the level 1 dose $(10 \mathrm{mg}$ of LEN) experienced progressive grade 2 SAEs and eventually dropped out. As shown in Table II, all 6 patients assigned to level 0 successfully completed at least 12 weeks of continuous administration of $5 \mathrm{mg}$ of LEN without any SAEs. Thus, continuous administration of LEN at $5 \mathrm{mg}$ may be acceptable in terms of safety, although that particular dose may be suboptimal considering the completion rate in our cohorts (i.e., $100 \%$ in the 5-mg cohort and 40\% in the 10-mg cohort). Therefore, it may be hypothesized that continuous administration of $7.5 \mathrm{mg}$ of LEN may be the next best dose; however, administration of $10 \mathrm{mg}$ of LEN for 21 out of every 28 days may represent a more realistic approach, as a 2.5-mg capsule form is not currently commercially available, and prolonged administration without drug holidays may raise concerns regarding the development of unexpected AEs. Although the number of patients in this study was inadequate for determining the response to therapy, none of the patients exhibited any evidence of disease progression or relapse while on LEN maintenance therapy.

In conclusion, our small study demonstrated that $5 \mathrm{mg}$ of LEN was acceptable in terms of safety, although maintenance therapy with $10 \mathrm{mg}$ of LEN administration for 21 out of every 28 days may be a more practical strategy for patients with MM following auto-PBSCT.

\section{Acknowledgements}

We would like to thank the nursing staff in all the participating transplant centers for their assistance in the collection of samples from patients included in this study. We are also grateful to the staff in all the participating transplant centers for their excellent patient care.

\section{References}

1. Kumar SK, Rajkumar SV, Dispenzieri A, Lacy MQ, Hayman SR, Buadi FK, Zeldenrust SR, Dingli D, Russell SJ, Lust JA, et al: Improved survival in multiple myeloma and the impact of novel therapies. Blood 111: 2516-2520, 2008.

2. Brenner H, Gondos A and Pulte D: Recent major improvement in long-term survival of younger patients with multiple myeloma. Blood 111: 2521-2526, 2008.
3. Alexanian R, Delasalle K, Wang M, Thomas S and Weber D: Curability of multiple myeloma. Bone Marrow Res 2012: 916479, 2012.

4. Attal M, Harousseau JL, Leyvraz S, Doyen C, Hulin C, Benboubker L, Yakoub Agha I, Bourhis JH, Garderet L, Pegourie B, et al: Maintenance therapy with thalidomide improves survival in patients with multiple myeloma. Blood 108: 3289-3294, 2006

5. Morgan GJ, Gregory WM, Davies FE, Bell SE, Szubert AJ, Brown JM, Coy NN, Cook G, Russell NH, Rudin C, et al: The role of maintenance thalidomide therapy in multiple myeloma: MRC Myeloma IX results and meta-analysis. Blood 119: 7-15, 2012.

6. McCarthy PL, Owzar K, Hofmeister CC, Hurd DD, Hassoun H, Richardson PG, Giralt S, Stadtmauer EA, Weisdorf DJ, Vij R, et al: Lenalidomide after stem-cell transplantation for multiple myeloma. N Engl J Med 366: 1770-1781, 2012.

7. Attal M, Lauwers-Cances V, Marit G, Caillot D, Moreau P, Facon T, Stoppa AM, Hulin C, Benboubker L, Garderet L, et al: Lenalidomide maintenance after stem-cell transplantation for multiple myeloma. N Engl J Med 366: 1782-1791, 2012.

8. Richardson PG, Schlossman RL, Weller E, Hideshima T, Mitsiades C, Davies F, LeBlanc R, Catley LP, Doss D, Kelly K, et al: Immunomodulatory drug CC-5013 overcomes drug resistance and is well tolerated in patients with relapsed multiple myeloma. Blood 100: 3063-3067, 2002.

9. Durie BG, Harousseau JL, Miguel JS, Bladé J, Barlogie B, Anderson K, Gertz M, Dimopoulos M, Westin J, Sonneveld P, et al: International uniform response criteria for multiple myeloma. Leukemia 20: 1467-1473, 2006.

10. O'Quigley J, Pepe M and Fisher L: Continual reassessment method: A practical design for phase 1 clinical trials in cancer. Biometrics 46: 33-48, 1990.

11. Ishizuka N and Morita S: Practical implementation of the continual reassessment method. Handbook of Statistics in Clinical Oncology, 2nd edition, 2006.

12. Palumbo A, Mina R, Cerrato $\mathrm{C}$ and Cavallo F: Role of consolidation/maintenance therapy in multiple myeloma. Clin Lymphoma Myeloma Leuk 13 (Suppl 2): S349-S354, 2013.

13. Abidi MH, Gul Z, Abrams J, Ayash L, Deol A, Ventimiglia M, Lum L, Mellon-Reppen S, Al-Kadhimi Z, Ratanatharathorn $\mathrm{V}$, et al: Phase I trial of bortezomib during maintenance phase after high dose melphalan and autologous stem cell transplantation in patients with multiple myeloma. J Chemother 24: 167-172, 2012.

14. Spencer A, Prince HM, Roberts AW, Prosser IW, Bradstock KF, Coyle L, Gill DS, Horvath N, Reynolds J and Kennedy N: Consolidation therapy with low-dose thalidomide and prednisolone prolongs the survival of multiple myeloma patients undergoing a single autologous stem-cell transplantation procedure. J Clin Oncol 27: 1788-1793, 2009.

15. Lokhorst HM, van der Holt B, Zweegman S, Vellenga E, Croockewit S, van Oers $\mathrm{MH}$, von dem Borne P, Wijermans $\mathrm{P}$, Schaafsma $\mathrm{R}$, de Weerdt $\mathrm{O}$, et al: A randomized phase 3 study on the effect of thalidomide combined with adriamycin, dexamethasone, and high-dose melphalan, followed by thalidomide maintenance in patients with multiple myeloma. Blood 115: 1113-1120, 2010.

16. Sonneveld P, Schmidt-Wolf IG, van der Holt B, El Jarari L, Bertsch U, Salwender H, Zweegman S, Vellenga E, Broyl A, Blau IW, et al: Bortezomib induction and maintenance treatment in patients with newly diagnosed multiple myeloma: Results of the randomized phase III HOVON-65/GMMG-HD4 trial. J Clin Oncol 30: 2946-2955, 2012.

17. Palumbo A, Cavallo F, Gay F, Di Raimondo F, Ben Yehuda D, Petrucci MT, Pezzatti S, Caravita T, Cerrato C, Ribakovsky E, et al: Autologous transplantation and maintenance therapy in multiple myeloma. N Engl J Med 371: 895-905, 2014. 